\title{
Reines Wismut.
}

\author{
Von F. Mruius und E. Groschuff. \\ (Mitteilung aus der Physikalisch-Technischen Reichsanstalt.)
}

Mit 1 Figur in Text.

Einleitung.

Übersicht。

A. Präparative Herstellung von reinem Wismut. Verfahren von SChNeIDER. - Kristallisation vom Nitrat $\mathrm{Bi}\left(\mathrm{NO}_{3}\right)_{3}+5 \mathrm{H}_{2} \mathrm{O}$. - Mischkristalle mit anderen Nitraten. - Trennung von vielen Verunreinigungen; Trennung vom Kupfer, Silber, Blei, Zinn usw. - Uberführung in Metall. Kristallisation von metallischem Wismut - Schmelzpunktserniedrigung durch Fremdstoffe; Schmelzpunktserhöhung durch Antimon. - Vollendung der Reinigung.

B. Analytische Untersuchung der Verunreinigungen: I. Qualitative Prüfung; II. Quantitative Analyse von nominell reinem Wismut: a) Auflösung und Kristallisation des Nitrats; b) Analyse des ungelösten Rückstandes; c) Analyse der unreinen Nitrat-Mutterlauge.

C. Chemische und physikalische Charakterisierung von Wismutmetall. 1. die chemische Analyse; 2. der Schmelzpunkt; 3. der elektrische Widerstand.

D. Ergebnis der Untersuchung.

\section{Einleitung.}

Das elementare Wismut ist gegenwärtig der Gegenstand mannigfacher Experimentalstudien, welche die Erforschung seiner allotropen Zustände zum Ziel haben ${ }^{1}$; hier ist die größte Reinheit des Materials erforderlich. In der Physik dient das Metall vielfach zu magnetischen und elektrischen Messungen, bei welchen es ebenfalls auf eine große Reinheit ankommt; auch bei der Verwendung des Metalls als Fixpunkt in der Thermometerskala ist man auf große Reinheit angewiesen. Absolut reines Wismut ist nicht zu beschaffen. Das im Handel zur Verfügung stehende nominell reine Motall bedarf einer sorgfältigeren Charakterisierung als ihm häufig

1 Vgl. Cohen und Moesveld, Zeitschr. phys. Chem. 85 (1913), 419. 
zuteil wird. Die Bemerkung: „In dem Präparat konnte keine Verunreinigung aufgefunden werden" erklärt sich gewöhnlich aus der Anwendung der duldsamen Vorschriften zur chemischen Prüfung des rohen Handelswismuts, welche zur Beurteilung reinerer Präparate nicht geeignet sind. Zu deren Charakterisierung bedarf es analytischer Methoden von größerer Empfindlichkeit. Die gegenwärtige Mitteilung soll dieser Frage näher kommen im Sinne vorangegangener Untersuchungen ${ }^{1}$, welche die analytische Feststellung verschiedener Reinigungsstufen bei den Metallen Blei, Zink, Cadmium, Zinn, Gold, Platin etc. zur Aufgabe hatten.

Während es je nach der Verwendung des Wismuts nahe liegt, einzelnen Verunreinigungen ein besonderes Gewicht beizulegen (z. B. dem Arsen für den pharmazeutischen Gebrauch, dem Zinn bei elektrischen Messungen, dem Kupfer bei Phosphoreszenzerscheinungen), wird der Reinheitsgrad, vom analytischen Standpunkt betrachtet, durch alle Fremdstoffe in gleicher Weise nach Maßgabe ihres Massenbetrages beeinflußt. Bei der chemischen Beurteilung wird man aber geneigt sein, die leicht zu beseitigende Verunreinigung mit Sauerst off zunächst außer Acht zu lassen.

$\mathrm{Als}$ häufige Verunreinigungen im gereinigten Wismutmetall kommen vorwiegend in Betracht die Metalle Silber, Blei, Kupfer, Eisen, Nickel, Zink, Arsen, Antimon, Zinn, neben welchen (meist in Spuren) noch die Elemente Platin, Gold, Silicium, Kohlenstoff, Schwefel, Tellur, Thallium und viele andere vorhanden sein können.

Wenn das Bedürfnis nach einer zweckmäßigen Vorschrift für die Aufsuchung oder Bestimmung sehr kleiner Verunreinigungen befriedigt werden soll, muß man berücksichtigen, daß hier die Hauptschwierigkeit darin besteht, aus einer Lösung das Wismut selbst in Form einer Verbindung - frei von Verunreinigungen abzusondern, so daß die letzteren im Filtrat konzentriert und bestimmt werden können. Auf andere Weise kann die Gesamtverunreinigung nicht ermittelt werden.

Die gewöhnliche analytische Methode zur Fällung des Wismuts als Oxyd oder Sulfid ist zu diesem Zwecke nicht geeignet, selbst wenn eine nachträgliche Behandlung mit Schwefelnatrium, Cyankalium usw. exfolgt.

Ehensowenig können andere amorphe Niederschläge in Betracht:

1 Vgl. F. Myuros, Z. anorg. Chem. 74 (1912), 407. 
kommen, weil sie stets Verunreinigungen einschlieBen. Wirksamer ist schon die Fällung des Wismuts als schwer lösliches kristallisches Oxychlorid, welches vielfach analytisch benutzt wird. Es ist jedoch daran zu erinnern, daß das aus einer salzsauren Wismutlösung durch Wasser gefällte Oxychlorid sich am Lichte meist dunkel färbt, und man kann sich leicht davon überzeugen, daß diese Färbung durch Chlorsilber veranlaBt wird, welches mit dem Wismutoxychlorid zur Abscheidung gelangt. Auch das Blei wird nebst anderen Verunreinigungen zum Teil mitgefällt.

Diese Mitfällung des Silbers und Bleies fällt fort, wenn man chlorfreie Nitratlösung benutzt und mit Wasser einen Niederschlag von basischem Nitrat erzeugt. Aber abgesehen von der Schwierigkeit, das Chlor fernzuhalten, mangelt es auch hier nicht an Fehlerquellen. So weiß man z. B., daB neben anderen Verunreinigungen ein etwaiger Arsengehalt meist in das basische Nitrat mit übergeht. Ebenso wird dabei die Mitfällung aller kolloidartigen Stoffe und im besonderen der Zinnsäure begünstigt, welche ihrerseits dann wieder bestimmte Verunreinigungen (Eisen, Kupfer usw.) mit einschließt. Man findet daher in den käuflichen Präparaten von basischem Wismutnitrat auch fast immer solche kolloide Verunreinigungen in kleinen Mengen. So z. B. hinterließen nach unseren Versuchen $100 \mathrm{~g}$ des ,Bismutum subnitricum purissimum" von.Schuchardt bei der Extraktion mit 10\% iger Salpetersäure $0.04 \mathrm{~g}$ eines dunklen Rückstandes, in welchem neben Zinnsäure auch Kieselsäure, Eisenoxyd, Silber usw. enthalten waren. Auch die Fällung als basisches Nitrat ist daher für die vorliegenden analytischen Zwecke. nicht zu empfehlen.

Endlich kann auch die elektrolytische Abscheidung als metallisches Wismut hier nicht in Betracht kommen wegen der Gefahr einer Verunreinigung mit anderen Metallen (z. B. Silber, Kupfer, Platin usw.) aus den Lösungen oder dem Material der Elektroden.

Man gelangt daher zu dem Schlusse, daß die Beseitigung der Hauptmenge des Wismuts aus der Lösung in schwerlöslicher Substanz behufs genauerer Analyse nicht einwandfrei durchzuführen ist.

Die analytische Kontrolle von nominell reinem Wismut kann nur erfolgen unter Berücksichtigung der Frage nach der präparativen Reinigung des Metalls auf nassem Wege, über welche bereits viele wertvolle Untersuchungen vorliegen. 


\section{A. Präparative Methode zur Herstellung von reinem Wismut.}

Das Wismut gehört zu denjenigen Metallen, welche leicht auf die zweite Reinigungsstufe gebracht werden können; die Verunreinigungen im technischen Wismut betragen meist weniger als $1 \%$. Eine sehr weitgehende Reinigung bietet hier aber die gleichen Schwierigkeiten wie bei den meisten anderen Metallen.

Bei Erörterung der Frage, welches Verfahren zur Herstellung von ganz reinem Wismut am geeignetsten ist, wird von manchen Autoren der rein chemische Weg, von anderen der elektrolytische Weg mit Eifer verfochten. Eine Entscheidung darüber kann im Grunde nur von statistischen Vergleichen erwartet werden, welche ein umfangreiches Versuchsmaterial nötig machen. Wir haben die Frage zu klären versucht und möchten unsere Erfahrungen im folgenden mitteilen.

Von rein chemischen Methoden interessiert uns hier wesentlich das Verfahren von SchNeIDER ${ }^{1}$, welcher als Ausgangsmaterial das leicht herstellbare kristallisierbare normale Nitrat $\mathrm{Bi}\left(\mathrm{NO}_{3}\right)_{3}+5 \mathrm{H}_{2} \mathrm{O}$ benutzt hat. Dasselbe ist jetzt ein leicht zugänglicher Handelsartikel. Nach Schneider wird das Nitrat durch Ausfällen der sauren Lösung mit Wasser partiell in basisches Salz übergeführt, welches nach dem Glühen Oxyd und bei der Roduktion mit Wasserstoff Wismutmetall ergibt, ,dessen metallische Verunreinigungen böchstens $0.01 \%$ betragen". Aus diesem Nachsatz geht hervor, daB der Autor eine solche Verunreinigung des Fällungsproduktes für unerheblich hält gegenüber der gröberen Verunreinigung des Ausgangsnitrates.

I. Moser ${ }^{2}$ fand bei der Nachprüfung die Angaben von Schneider bestätigt und hebt hervor, daß in dem bei zweimaliger partieller Fällung (als basisches Salz) hergestellten Wismut auf spektralanalytischem Wege kein anderes Metall nachgewiesen werden konnte.

Trotz der oben hervorgehobenen Fehlerquellen bei der ausgiebigen hydrolytischen Zersetzung unreinen Wismutnitrates bestätigen unsere eigenen Versuche ebenfalls die Beobachtungen von Schneider und Moser, daß man durch partielle t'ällung einer Wismutnitratlösung mit Wasser (wobei relativ saure Verbindungen entstehen) zu Niederschlägen von großer Reinheit gelangen kann, besonders, wenn die Nitratlösung selbst schon gereinigt ist.

1 Schnamer, Journ. f. prakt. Chem. [N. F.] 44 (1891), 41.

${ }^{2}$ L. Moser, Die Bestimmungsmethoden des Wismuts usw. Stuttgart 1909. 
Wenn man aber die Forderung von Moser befolgt, die partielle Fällung zu wiederholen, so wird die Ausbeute an dem kristallischen Produkt so unbefriedigend, daß das Verfahren für präparative Zwecke kaum noch rationell erscheint. Offenbar wird hier wie bei SchNeIDER noch eine gröbere Verunreinigung des Ausgangsnitrates vorausgesetzt. Es fragt sich aber, ob es in diesem Falle nicht zweckmäßiger ist, die vollständige Reinigung durch wiederholte Kristallisation des normalen Salzes selbst aus saurer Lösung (bei Vermeidung der hydrolytischen Zersetzung) herbeizuführen. Unsere Versuche darüber hatten insofern Erfolg, als es leicht gelang, die Wismutnitratlösungen mit konzentrierter Salpetersäure (anstatt mit Wasser) auszufällen und so bei kleinem Volumen der Lösungen gute Ausbeuten an reinen Kristallen von normalem Wismutnitrat zu erhalten.

Dieser Reinigungsart kommt die trikline Form und die ungewöhnliche Zusammensetzung des Salzes $\mathrm{Bi}\left(\mathrm{NO}_{3}\right)_{3}+5 \mathrm{H}_{2} \mathrm{O}$ zustatten, welche bei den Nitraten der übrigen Schwermetalle auch kristallographisch kaum ein Analogon findet. Die Fähigkeit, isomorphe Mischkristalle zu bilden, ist demnach gering. Immerhin ist sie aber mit Sicherheit durch BoDmanN ${ }^{1}$ nachgewiesen zwischen dem Wismutnitrat und den Nitraten der seltenen Erdmetalle Didy m, Lanthan und Yttrium, welche damit zwei Reihen isomorpher Mischkristalle mit 5 und $6 \mathrm{H}_{2} \mathrm{O}$ bilden.

Wie mit den Nitraten der seltenen Erden ist ferner auch das Wismutnitrat befähigt, kristallisierbare (aber damit nicht isomorphe) Joppelsalze mit den Nitraten von Magnesium, Zink, Mangan, Nickel und Kobalt zu bilden, welche dem allgemeinen Typus $3 \mathrm{Me}\left(\mathrm{NO}_{3}\right)_{2}, 2 \mathrm{Bi}\left(\mathrm{NO}_{3}\right)_{3},+24 \mathrm{H}_{2} \mathrm{O}$ angehören. ${ }^{2}$

Während die seltenen Erden als Verunreinigungen des Wismuts nicht in Betracht kommen, ist dies wohl mit einigen Metallen der Zinkreihe der Fall. Die erwähnten Doppelnitrate sind jedoch so leicht löslich und zersetzlich, daß sie sich nur bei großer Konzentration beider Komponenten bilden können, nicht aber, wenn in einer übersättigten Wismutnitratlösung nur Spuren der fremden Metallnitrate anwesend sind. Ein Versuch mit dem Nickel (als Vertreter der Zinkgruppe) ergab folgendes Verhalten.

$9.6 \mathrm{~g}$ Wismutnitrat und $9.3 \mathrm{~g}$ Nickelnitratkristalle konnten zusammengerieben werden, ohne daß Verflüssigung stattfand. Eine

1 G. Bodmans, Ber. deutsch. Chem. Ges. 31 (1898), 1237.

2 Urbatn und Lacombe, Compt. rend. 137 (1903), 568. 
solche trat aber bei leichter Erwärmung ein. Bei der Abkühlung der klaren Lösung erfolgte die Erstarrung zu blaßgrünen Kristallen des Doppelsalzes, welches sich mit Wasser unter weißer Trübung zersetzt.

Wird jedoch die Verbindung in wenig warmer verdünnter Salpetersäure gelöst und die überkaltete Lösung mit einem Kriställchen von Wismutnitrat versetzt, so erhält man zunächst eine reichliche Abscheidung ganz farbloser Wismutnitratkristalle ohne Nickelgehalt, und bei weiterer Abkühlung dunkelgrüne Kiristalle von Nickelnitrat, welche wismutfrei sind und sich ohne Trübung in Wasser lösen. Die Verwandtschaft zwischen den beiden Komponenten ist also sehr gering und schon durch einen Überschuß von Salpetersäure zugunsten der reinen Komponenten aufzuheben.

Bei dem Umkristallisieren des Wismutnitrats aus unreinen Lösungen ist denn auch von einer Absorption fremder Nitrate durch Bildung von Mischkristallen, Doppelsalzen oder dergleichen nichts zu bemerken, ganz im Sinne der früheren Beobachtungen von Schneider. Da es aber einige Metallnitrate sowie oxydische Stoffe gibt, welche in Salpetersäure schwerer löslich sind als das Wismutnitrat, so ist die strenge Forderung, sämtliche Verunreinigungen desselben durch Extraktion zu beseitigen, nur in bedingter Weise zu erfüllen.

Bei dem folgenden gröberen Versuch wird die Reinigung zunächst durch Fällung der Fremdstoffe und dann durch Extraktion derselben herbeigeführt.

\section{Trennang von vielen Verunreinigungen.}

$\mathrm{Zu}$ einer Iösung von $32 \mathrm{~g}$ Wismutnitrat in $16 \mathrm{~g} 8 \%$ iger Salpetersäure wurden die Nitrate von je einem I)ezigramm der acht Metalle Silber, Kupfer, Blei, Eisen, Nickel, Zink, Cadmium und Quecksilber gefügt. Ferner brachte man dazu eine kalte Lösung von je einem Dezigramm Arsen, Antimon und Zinn in stark verdünnter Salpetersäure. Der alsbald auftretende blaßgelbe Niederschlag vermehrte sich noch während einer einstündigen Erwärmung. I)as nach dem Erkalten erhaltene klare grüne Filtrat von $50 \mathrm{~g}$ wurde mit dem gleichen Gewicht konzentrierter Salpetersäure versetzt und bei $0^{0}$ zur Kristallisation angeregt. Nach gutem Absaugen und Waschen mit kalter Salpetersäure wurden völlig farblose Wismutnitratkristalle erhalten, welche nur noch Spuren der Verunreinigungen enthielten. Durch einmaliges Umkristallisieren aus ver- 
dünnter Salpetersäure gelang die Reinigung so weit, daß (in kleinen Massen) kein fremdes Metall mehr direkt nachzuweisen war.

\section{Einzelne Verunreinigangen.}

Abgesehen von den durch direkte Fällung abgeschiedenen Fremdstoffen darf man wohi annehmen, da $B$ bei dem Umkristallisieren des unreinen Wismutnitrats der Übergang der Verunreinigungen in die Mutterlauge nahezu gleichförmig nach MaBgabe ihres Massenbetrages erfolgt. In diesem Sinne kann man den Gesamteffekt der Reinigung an dem Verschwinden einer einzelnen Verunreinigung (z. B. $\mathrm{Cu}, \mathrm{Ag}, \mathrm{Pb}$ usw.) verfolgen.

Kupfer. Zur kolorimetrischen Beobachtung eignet sich besonders gut das Kupfer, welches wohl im Handelswismut niemals ganz fehlt. Bei dem Umkristallisieren des unreinen Nitrats sind die Mutterlaugen immer schwach aber deutlich grün gefärbt, während die Kristalle farblos erscheinen. Wenn man die Kristallisation aus Salpetersäure so lange fortsetzt, bis auch die auf ein sehr kleines Volumen gebrachte Mutterlauge völlig farblos erscheint und nach dem Eindampfen auch mit Ammoniaklösung keinen bläulichen Auszug mehr ergibt, so kann man dies als einen Beweis weitgehender Reinigung der Kristalle betrachten. In diesem Zustand der Reinheit erhält man das Wismutnitrat auch bei der ersten Kristallisation, wenn es im Ausgangsmaterial mit der gleichen Menge Kupfernitrat gemischt ist.

Silber. Die fortschreitende Reinigung durch Extraktion zeigt die folgende Versuchsreihe.

$100 \mathrm{~g}$ Wismut wurden mit $2 \mathrm{~g}$ Silber in $250 \mathrm{~g}$ konzentrierter Salpetersäure (1.40) gelöst. Bei dem Erkalten der Lösung erhielt man $140 \mathrm{~g}$ kristallisiertes Nitrat I, welches gemäß der Analyse, bezogen auf das vorhandene Wismut, $0.29 \%$ Silber enthielt. Die bei dem Umkristallisieren mit 35 g Salpetersäure erhaltenen Kristalle II ergaben $0.06 \%$, und in den bei nochmaligem Kristallisieren (mit $35 \mathrm{~g}$ Salpetersäure) gewonnenen Kristallen III fand sich nur noch $0.008 \%$ auf Wismut bezogenes Silber, während es im Ausgangsmaterial $2 \%$ betragen hatte. Mithin war im Wismutsalz durch dreimaliges Umkristallisieren die prozentische Verunreinigung auf den 250. Teil vermindert worden, und die gereinigten Kristalle enthielten in der Gewichtseinheit nur $\mathbf{0 . 4 \%}$ der anfänglichen Verunreinigung. Die Ausbeute der gereinigten Substanz betrug $107 \mathrm{~g}$ Nitrat entsprechend $46 \%$ des Ansgangsmaterials; die Mutterlaugen waren nur durch Abgießen entfernt worden. 
Blei. Eine besondere Besprechung verlangt die Verunreinigung mit Blei, welches in käuflichen Wismut immer vorhanden ist.

1)ie Löslichkeit des Bleinitrats wird durch Salpetersäure von steigender Konzentration in viel höherem Grade herabgedrückt als dies bei dem Wismutnitrat der Fall ist. Die gesättigte Lösung enthält bei Anwendung von

\begin{tabular}{l|c|c}
\hline \hline Lösungsmittel & $\mathrm{Bi}\left(\mathrm{NO}_{3}\right)_{3}+5 \mathrm{H}_{2} \mathrm{O}$ & $\mathrm{Pb}\left(\mathrm{NO}_{3}\right)_{2}$ \\
\hline \hline Wasser & zersetzt & etwa $35 \%$ \\
$20 \%$ Salpetersäure & etwa $62 \%$ &, $6 \%$ \\
$40 \%$ & $\#, 42 \%$ & $\# 1.4 \%$ \\
$65 \%$ & $\#, 025 \%$ &, $0.05 \%$
\end{tabular}

Auch für das bleihaltige Wismut bleibt dies Verhalten der Salpetersäure sinngemäß bestehen. Als Lösungsmittel für die reinigende Kristallisation ist hier verdünnte Salpetersäure anzuwenden, nicht aber konzentrierte, welche das Bleinitrat mit ausfällt. Man kann von diesem letzteren Vorgang Anwendung machen, um aus stark bleihaltigem Wismutnitrat zunächst die Hauptmenge des Bleies durch Fällung zu beseitigen, während man das Wismut in Lösung hält. Der Abdampfrückstand läßt sich dann leicht von dem noch vorhandenen kleinen Bleigehalt durch Umkristallisieren aus verdünnter Salpetersäure befreien. 1)er Bleigehalt des Wismutnitrats darf einen (für Salpetersäure verschiedener Konzentration wechselnden) Betrag nicht überschreiten, wenn er in die Mutterlauge übergehen, und die Reinigung der Kristalle gelingen soll; wird hier die Sättigungsgrenze für das Bleinitrat überschritten, so tritt Fällung ein.

Bei Wismutnitrat mit 1\% Bleinitrat läßt sich die Kristallisation mit. stärkerer Salpetersäure nicht mehr befriedigend durchführen, weil das Bleinitrat mit ausfällt.

I)as nominell reine Wismutmetall des Handels enthält gewöhnlich weniger Blei als dieser schädlichen Grenze $(0.2$ bis $1 \%$ ) entspricht.

Wismutnitrat, welches $0.5 \%$ Bleinitrat enthielt, konnte durch fünfmalige Kristallisation aus $20 \%$ iger Salpetersäure bis zur Grenze der Nachweisbarkeit vom Blei befreit werden; aber auch bei einmaligem Lmkristallisieren aus $2 / 3$ seines Gewichtes $40 \%$ iger Salpetersäure ergab das unreine Ausgangsmaterial klare gut entwickelte Kristalle von Wismutnitrat, in welchen kaum eine Spur Blei nachgewiesen werden konnte, während die Mutterlauge reich daran war. 
Die Versuche ergeben, daß sich eine klein $\Theta$ Verunreinigung des Wismutnitrats mitBleinitrat leicht durch Kristallisa tion (Extraktion) entfernen läßt; ein großer Bleigehalt wäre zuvor mit konzentrierter Salpetersäure auszufällen. Aber auch die Elektrolyse einer sauren Wismutnitratlösung kann dazu dienen, sie (durch Abscheidung von Superoxyd an der Platinanode) soweit vom Blei zu befreien, daß bei der späteren Kristallisation eine Veruureinigung des Wismutnitrats mit Bleinitrat gänzlich ausgeschlossen ist. Die Löslichkeit der übrigen schädlichen Fremdstoffe wird bei zunehmender Konzentration der Salpetersäure (unähnlich dem Bleinitrat) in der Regel vermehrt; dies gilt z. B. auch für das Chlorsilber, welches bei einem Chlorgehalt der Salpetersäure leicht als Verunreinigung auftreten kann. Arsen, welches als Wismutarseniat - und Antimon, welches als Antimoniat zur Abscheidung gebracht wird, gehören ebenfalls dahin.

Zinn. Das Zinn gelangt bei der Auflösung unreinen Wismuts in konzentrierter Salpetersäure größtenteils als zinnsaures oder metazinnsaures Wismut (mit Silber, Kupfer, Eisen usw.) zur Abscheidung. Ein sehr kleiner Teil bleibt jedoch gelöst und wird erst bei längerem Erwärmen der mit Wasser verdünnten Lösung als leichte Trübung abgeschieden (welche sich auch in konzentrierter Salpetersäure kaum noch löst). Die abfiltrierte Lösung ist nunmehr in der Wärme noch mit Spuren von Metazinnsäure ,gesättigt". Durch starke Abkühlung unter Zusatz konzentrierter Salpetersäure kann die Sättigung aber aufgehoben werden. Man erhält dann reines Wismutnitrat in Kristallen, während die extrahierten Spuren noch vorhandener Zinnsäure in aie Mutterlauge übergehen.

Die endgültige Kristallisation bei der präparativen Reinigung des normalen Wismutnitrats wird demnach in jedem Fall so vorgenommen, daß man die schon sehr reinen Salzkristalle in der Hälfte ihres Gewichts $8 \%$ iger Salpetersäure bei etwa $18^{\circ}$ löst (wobei sich keine Trübung von Zinnsäure zeigen darf) und die Lösung mit dem gleichen Gewicht konzentrierter Salpetersäure (1.40) vermischt. Bei der Abkühlung auf 0 bis $-10^{\circ}$ und geringer Bewegung erstarrt dann die völlig klare Lösung zu einem Kristallbrei, aus welchem sich das Salz durch Absaugen und Waschen mit wenig eiskalter Salpetersäure leicht im reinsten Zustande isolieren läBt.

Die vorstehenden Versuche sollten für die präparativen Zwecke den Nachweis führen, daB sich das normale Wismutnitrat durch Kristallisation aus Salpetersäure unbegrenzt reinigen läßt, 
so daß es auch bei Vermeidung der Fällung mit Wasser als Grundlage für die Herstellung von reinem Wismutmetall dienen kann.

Andererseits kommt diese Tatsache auch der analytischen Richtung zustatten, welche auf den Nachweis sehr kleiner Verunreinigungen ausgeht. 1)a man diese einwandfrei in den Mutterlaugen aufspeichern kann, werden sie dadurch auch bei spurenweisem Vorkommen der direkten Bestimmung leicht zugänglich.

Die in einem späteren Abschnitt beschriebene Methode zur analytischen Charakterisierung von reinem Wismutmetall gründet sich auf die vorstehend besprochene Nitratkristallisation.

\section{Überführung in Metall.}

Soll das reine Wismutnitrat präparativ auf Metall verarbeitet werden, so benutzt man das basische Nitrat und das Oxyd als Übergangsprodukte. Zur Herstellung derselben erscheint der nasse Weg überflüssig. Das kristallisierte Nitrat wird in einer Porzellanschale unter beständigem Rühren auf etwa $110^{\circ}$ erwärmt, wobei es unter Abgabe von Salpetersäure zunächst zu einer durch basisches Salz getrübten Flüssigkeit schmilzt; dieselbe verdickt sich mehr und mehr, bis zuletzt ein trockener Rückstand von basischem Nitrat übrig bleibt. Durch Glühen desselben erhält man das in der Hitze braune und bei Zimmertemperatur zitronengelbe Wismutoxyd.

Das Oxyd läßt sich in schwer schmelzbaren Glasröhren durch Glühen im Wasserstoff zu Metall reduzieren. Bequemer ist aber die Reduktion durch Zusammenschmelzen des Wismutoxyds mit seinem halben Gewicht Cyankalium in Porzellantiegeln. ${ }^{1}$

Der Reinheitsgrad des auf diese Weise mit Sorgfalt hergestellten Wismutmetalls wird so hoch sein, daß die Verunreinigungen analytisch nicht mehr definierbar sind. Dennoch ist auch hier eine absolute Reinheit keineswegs anzunehmen. Zu möglichster Annäherung an diesen idealen Fall ist noch eine weitere Reinigung des Wismuts im metallischen Zustand in Betracht zu ziehen. An eine reinigende Wirkung der Destillation ist hier nicht zu denken, da das Wismut schwerer als die meisten seiner Verunreinigungen flüchtig ist und erst jenseits $1500^{\circ}$ siedet. Dagegen kann hier zunächst das Umschmelzen des Metalls an der Luft reinigend wirken, indem es durch Oxydation (in Suspension oder Lösung befind-

1 Vgl. darüber Treadweld, Ann. Chem. II, 133, sowie Egank, Zeitschr. phys. Chem. 64 (1908), 489. 
liche) „verbrennliche Stoffe" gasförmig oder als Schlacke zur Abscheidung bringt.

Vor allem scheint aber die Kristallisation aus dem Schmelzfluß für die endgültige Reinigung des Wismuts zweckmäßig.

\section{Kristallisation von motallisohem Wismut.}

Bei dem hervorragenden Kristallisationsvermögen des Wismuts ist es schon längst festgestellt worden, daß bei dem langsamen Hrstarren des geschmolzenen Metalls die zunächst (unter Volumzunahme) sich abscheidenden Kristalle sehr rein sind. Die Verunreinigungen gehen in die metallische „Mutterlauge“ über, deren zuletzt erstarrende Teile als Wülste über die Oberfläche hinausgedrängt werden (WinkLer-Scuneider). In der Technik macht man daher von der Kristallisation des Wismuts schon seit langer Zeit praktische Anwendung. Die im Handel vorkommenden Wismutkristalle sind mehr oder weniger rein, entsprechend der Natur des Ausgangsmaterials und der angewandten Sorgfalt bei der Kristallisation. In den Handelspräparaten (wie z. B. in dem uns vorliegenden Kristallwismut von SchuchardT) sind die gewöhnlichen Verunreinigungen des Wismuts aber immer noch in nachweisbaren Spuren vorhanden.

Wissenschaftlich sind in neuerer Zeit die "Schmelzdiagramme“ der Wismutlegierungen eingehend studiert worden. Zur Orientierung über die maximale Schmelzpunktserniedrigung des Wismuts durch Verunreinigungen möge die folgende Übersicht dienen, in welcher die wichtigsten Beobachtungen der letzten Jahre über die Lage des Futektikums verzeichnet sind.

Aus umstehender Tabelle sind die großen Unterschiede in der Konzentration der Fremdstoffe im Eutektikum zu erkennen, welche für die Lage desselben im Zustandsdiagramm maßgebend ist; außer dieser ist für die Reinigung des Wismuts durch Kristallisation auch die Bildung von Mischkristallen von Bedeutung. $\mathrm{Hg}, \mathrm{Sn}, \mathrm{Cd}, \mathrm{Fe}$, $\mathrm{Au}, \mathrm{Na}$ zeigen eine sebr niedrig gelegene eutektische Temperatur; die Trennung von den Wismutkristallen wird hier also leicht erfolgen. Das gleiche ist bei $\mathrm{Pb}$ und $\mathrm{Zn}$ der Fall. Der Reinigungseffekt ist hier aber etwas beschränkt durch die Bildung von Mischkristallen oberhalb der eutektischen Temperatur. Auch bei Co, Ag, $\mathrm{Ca}, \mathrm{K}$ und den Nichtmetallen $\mathrm{Br}, \mathrm{Cl}, \mathrm{J}, \mathrm{S}$, Li, für welche die Schmelzpunktsdifferenz des Eutektikums gegenüber dem reinen Wismut 2 bis $11^{\circ}$ beträgt, ist die Reinigung durch Kristallisation 


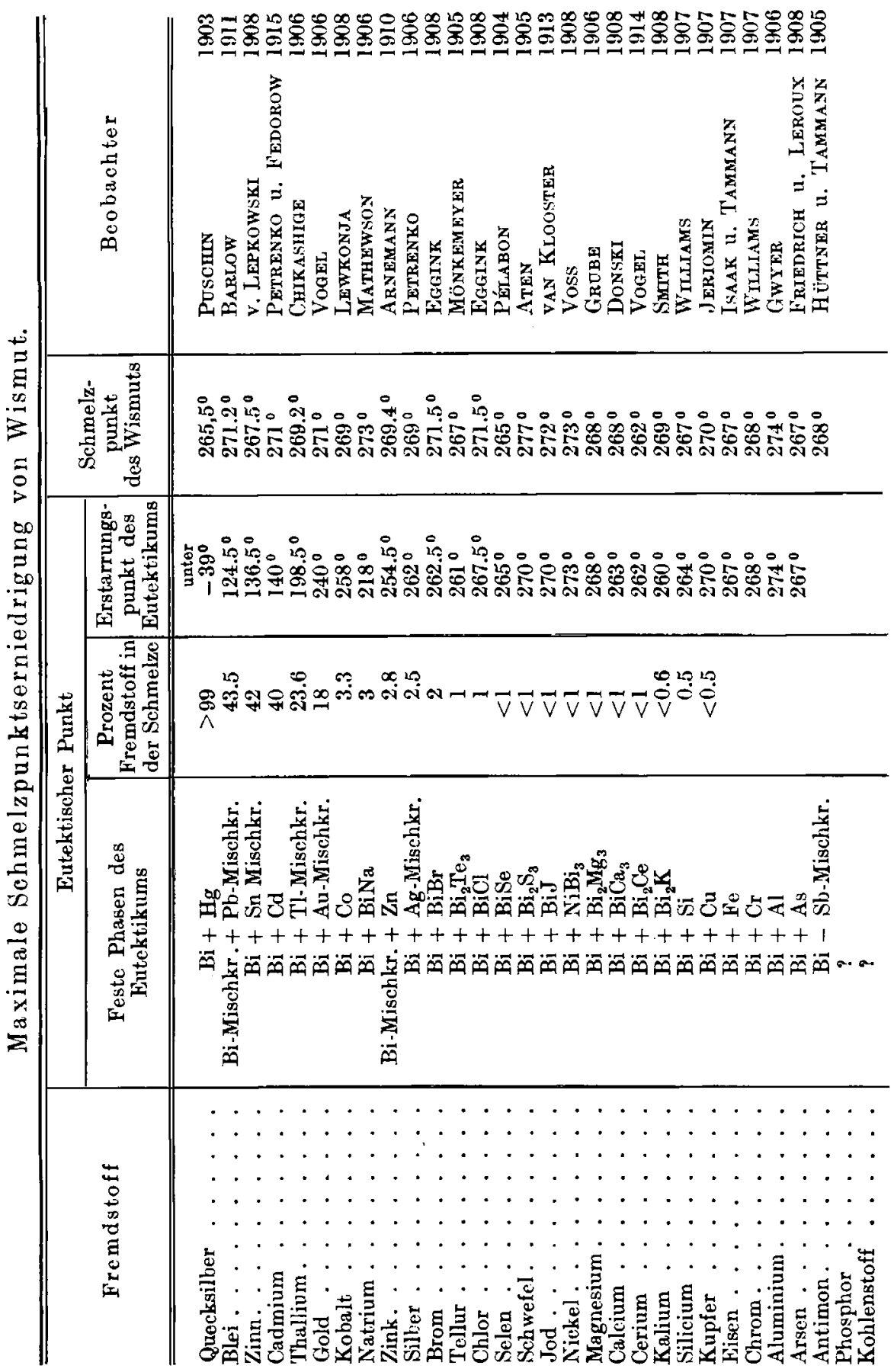


noch leicht durchführbar. Bei $\mathrm{Mg}, \mathrm{Cu}, \mathrm{Ni}$, Ce, Se sind die Fremdstoffe im Eutektikum in so kleiner Menge enthalten, daß die Tabelle keine Schmelzpunktsdifferenz gegen Wismut angibt; genauere $\mathrm{Be}$ obachtungen werden solche später vermutlich noch feststellen.

Das Prinzip der Reinigung des Wismuts durch Kristallisation aus dem Schmelzfluß erstreckt sich' sonach auf sehr zahlreiche Elemente; es würde allgemeingültig sein, wenn nicht auch ausnahmsweise der entgegengesetzte Fall vorkäme, daß der Zusatz eines Elements den Schmelzpunkt erhöht und demgemäß bei dem unvollständigen Erstarren der Metallschmelze eine Aufspeicherung der Verunreinigung in den Krystallen statt in der "Mutterlauge“ stattfindet. Ein solcher Fall liegt bei dem schwerer schmelzbaren, aber im Wismut leicht löslichen Antimon vor, welches damit eine lückenlose Reihe isomorpher Mischkristalle ergibt; die Schmelzpunkte derselben nehmen mit wachsendem Antimongehalt zu.

Die Wirkung des wenig löslichen Phosphors, des Arsens und vieler seltenerer Elemente bedarf noch näherer Untersuchung. Kohlenstoff, Eisen, Chrom, Aluminium gelten als unlöslich. Bei derartigen schwer schmelzbaren Verunreinigungen bleibt die Schmelzpunktserniedrigung zweifelhaft. Auch die Löslichkeit des elementaren Sauerstoffs (als $\mathrm{BiO}$ ) ist kaum nachweisbar. Das Wismutoxydul wird durch Wasserstoff leicht reduziert; ein Sauerstoffgehalt würde also durch Glühen des Metalls im Wasserstoff an der Gewichtsverminderung leicht zu messen sein. $11.5 \mathrm{~g}$ des an der Luft geschmolzenen und im evakuierten Glasrohr erstarrten Metalls ergaben uns dabei nur einen Gewichtsverlust von $0.2 \mathrm{mg}$, wovon noch ein kleines Sublimat von Wismut in Abzug zu bringen ist. Die Löslichkeit des Sanerstoffs im Metall wird nahe der Schmelztemperatur wohl kaum $1 / 1000$ Gew.- $\%$ erreichen.

Als Beleg für die Wirksamkeit der Kristallisation aus dem Schmelzfluß haben wir die folgenden Versuche ausgeführt.

I. Wismut mit je $1 / 10 \%$ Zinn, Kupfer und Silber wurde ans dem SchmelzfluB der unvollständigen, zweimal wiederholten Kristallisation unterworfen, wobei der Zutritt der Luft durch eine Paraffinschicht abgehalten war.

Die isolierten Wismutkristalle ergaben bei längerem Erwärmen mit Salpetersäure eine klare zinnfreie Lösung und nach dem Übersättigen ein farbloses kupferfreies Filtrat, in welchem durch Salzsäure kaum noch eine Spur Silber nachzuweisen war. Die metallische Mutterlauge dagegen lieferte bei gleicher Be- 
handlung eine durch Zinnsäure stark getrübte Nitratlösung, sowie nach Zusatz von Ammoniak ein blaues kupferhaltiges Filtrat, in welchem durch Salzsäure ein starker Niederschlag von Chlorsilber erzeugt wurde.

II. Wismut mit einem Gehalt an $1 / 10 \%$ Antimon wurde der dreimal wiederholten Kristallisation wie bei I unterworfen. Jie Krystalle ergaben hier mit konzentrierter Salpetersäure zunächst eine klare Lösung, in welcher aber bei dem Erwärmen ein starker Niederschlag von Antimonsäure entstand; dagegen blieb die entsprechende Lösung der metallischen „Mutterlauge", auch bei längerer Erwärmung fast klar.

III. Ein gleichartiger Versuch mit Wismut, welches neben $0.1 \%$ Antimon noch $1 \%$ Blei enthielt, zeigte das gleiche Ergebnis wie Versuch II; die antimonhaltigen Wismutkristalle zeigten sich hier frei von Blei, die metallische Mutterlauge aber enthielt dasselbe, denn aus ihrer salzsauren Chloridlösung wurde durch Alkohol ein starker Niederschlag von Bleichlorid zur Abscheidung gebracht. An der trennenden Wirksamkeit der fraktionierten Kristallisation des metallischen Wismuts ist demnach nieht zu zweifeln; wenn dieselbe aber reinigend wirken soll, so muB das Antimon ${ }^{1}$ völlig abwesend sein, dessen Aufspeicherung in den Kristallen auch durch die Anwesenheit anderer Verunreinigungen nicht verhindert wird. Auch „unlösliche“" Fremdstoffe wie $\mathrm{Fe}$, Cr, Al, P usw. sollten ent. fernt sein, da eine Verunreinigung der Wismutkristalle mit diesen immerhin nahe liegt. Diese Forderungen sind bei dem.nach dem Nitratverfahren gereinigten Wismut zu erfüllen. Da aber auch bei diesem Metall eine absolute Reinheit nicht vorauszusetzen ist, so verspricht hier die Kristallisation aus dem Schmelzfluß noch eine ergänzende Reiniung, indem sie noch Spuren zahlreicher Fremdstoffe zu entfernen erlaubt, welche der Erkennung und Beseitigung auf nassem Wege entgangen sein können.

\section{Kristallisation von Wismut unter Paraffin.}

Die Operation wird zweckmäßig in weiten Probierröhren ausgeführt, in denen man bequem 200 bis $300 \mathrm{~g}$ Metall unter einer Decke von etwa $20 \mathrm{~g}$ Paraffin verarbeiten kann. Bei vorsichtigem Fächeln mit einer Bunsenflamme tritt während des Schmelzens

1 Nach Matriey läßt sich das Antimon durch bloßes Erhitzen des Wismutmetalls auf $458^{\circ}$ an der Luft als unlösliches $\mathrm{Sb}_{2} \mathrm{O}_{3}$ entfernen. Jahresbericht 1893, I, 378. 
und Kristallisierens gewöhnlich kein Zerspringen des Glasrohrs ein, leichter aber nach dem völligen Erstarren; eine untergestellte Schale darf niemals fehlen. Wird oxydhaltiges Wismut unter Paraffin geschmolzen, so findet eine scharfe Sonderung statt. Das ungelöste Oxyd wira vom Paraffin benetzt und färbt sich bei stärkerem Erhitzen schwarz. Nach dem Erkalten enthält die abgleöste Paraffinschicht die Oxyde in Suspension; das schlackenfreie Metall aber läBt sich von den noch anhaftenden ,Schmutzteilen" durch Abwaschen mit Äther und Putzen mit einem Tuch säubern. Zu bedenken ist noch, daß ein wenig Paraffin in etwaige bei der Abkühlung entstandene Risse eingedrungen sein kann.

Ein solehes reinigendes Umschmelzen des Metalls ist vor der eigentlichen Kristallisation sehr zu empfehlen. Die letztere wird gemäß Fig. 1 so durchgeführt, daß man das unter einer klaren Paraffinschicht geschmolzene Metall während der Abkühlung mit einem am Boden mit weiterem Loch versehenen und die Rohrweite nicht ganz ausfüllenden dünnen Löffel aus Glas in senkrechter Richtung bis zum Boden rührt; die dabei entstehenden Kristalle gelangen dabei durch Auftrieb nach oben und bleiben bei dem Emporheben des Löffels in diesem als loses Haufwerk liegen; das Abfließen des noch geschmolzenen Metallanteils kann man durch ruckweises Emporheben über die Paraffinschicht wesentlich befördern. ${ }^{1}$ Die Kristalle werden darauf in eine Porzellanschale geschüttet. Den so hergestellten reinen Wismutkristallen haftet noch etwas Paraffin

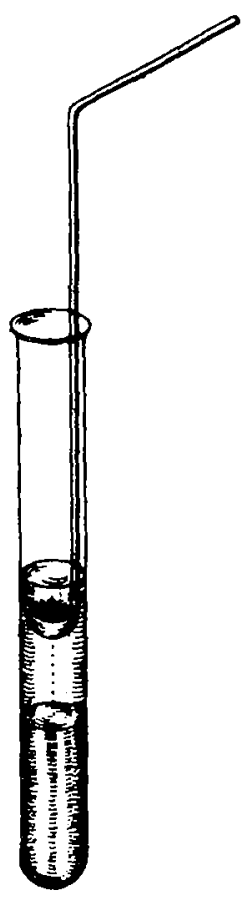

Fig. 1.

an. Man entfernt dasselbe durch Erhitzen im offenen Tiegel, wobei die organische Substanz verbrennt und das geschmolzene Metall sich oberflächlich oxydiert.

Das umgegossene noch flüssige Metall wird zuletzt in völlig evakuierte vorgewärmte Glasröhrchen von 5 bis $10 \mathrm{ccm}$ aufgesaugt, deren in eine Spitze ausgezogenes Ende man durch einen Druck auf den Tiegelboden zerbricht.

1 Bei der Arbeit in größerem Maßstabe würde eine Zentrifuge angebracht sein. 
Nach dem Abkühlen zeigt das vollkommen gefüllte Röhrchen gewöhnlich Risse, zwischen denen einige flüssige Metalltröpfchen hervorgequollen sind; diese lassen sich mit der Glasschicht leicht von dem spiegelblanken Metallzylinder entfernen.

Zur Herstellung reinen Wismuts hat sich demnach die folgende Reihe von Operationen als zweckmäßig erwiesen:

1. Kristallisation als normales Nitrat.

2. Glühen zu Oxyd.

3. Reduktion mit Cyankalium oder Wasserstoff.

4. Kristallisation des Metalls unter Paraffin.

5. Oberflächliche Oxydation.

6. Einsaugen in evakuierte Röhren.

Das so gewonnene Wismutmetall enthält als wahrscheinliche Verunreinigung noch eine Spur Sauerstoff, welche nötigenfalls durch Wasserstoff zu beseitigen ist.

\section{B. Analytische Untersuchung.}

\section{Qualitative Prüfung von Wismutmetall.}

Der Nachweis für die Reinheit (elementare Natur) eines Metalls ist nur indirekt auf chemischem Wege zu erbringen, während der physikalische Weg dazu nicht genügt. Die Verunreinigungen können sehr mannigfacher Art sein, und es bedarf zu ihrer Erkennung der verschiedensten chemischen Reaktionen. Auch wenn man diese erschöpft zu haben glaubt, bleibt noch der Einwand, unbekannte Fremdstoffe seien nicht berücksichtigt und bekannte ihrer kleinen Menge wegen übersehen worden. Nichts ist daher schwieriger als die genaue qualitative Charakterisierung einer vorliegenden Metallsubstanz. Die Prüfung läßt sich jedoch in Regeln bringen, welche je nach der angewandten Sorgfalt größeren oder geringeren Anforderungen an die Genauigkeit genügen.

Reines Wismut zeigt chemisch das folgende Verhalten.

Trockener Weg:

a) Vollkommene Löslichkeit in Quecksilber.

b) Bei dem Glühen in Wasserstoff kein Sublimat, keine Bildung von Wasser oder Schwefelwasserstoff.

c) Bei dem Glühen in Sauerstoff keine Bildung von Kohlensäure oder anderen flüchtigen Oxyden.

d) Bei dem Erhitzen im Chlorstrome vollkommene Flüchtigkeit. Produkt: weißes Wismutchlorid. 
Nasser Weg: Abwesenheit orkenpbarex

1. Vollkommene Löslichkeit in Salpeter- $\begin{aligned} & \begin{array}{c}\text { Mengen der Verunreinigungen } \\ \text { säure }\end{array} \\ & \text { timon, Arsen, Kohle, } \\ & \text { Schlackenbestandteile } \\ & \text { usw. }\end{aligned}$

$\left.\begin{array}{l}\text { 2. Keine Fällung der Nitratlösung mit } \\ \text { wenig Salzsäure }\end{array}\right\}$ Silber (Thallium).

3. Nach mehrfachem Eindampfen der Lösung 1 mit Salzsäure ergibt das Kupfer, Eisen, Nickel, Chlorid mit konzentrierter Salzsäure Kobalt usw. eine farblose Lösung

4. Lösung 3 ergibt auf Zusatz von Alkohol und wenig Schwefelsäure keine Trübung

5. Keine dunkle Färbung der salzsauren |Arsen, Quecksilber, Chloridlösung mit Zinnchlorür

6. Nach Ausfällen der verdünnten Salzlösungen mit Schwefelwasserstoff ist im sauren Filtrat kein fremdes Metall nachweisbar

7. Das gefällte Sulfid gibt an Schwefelammonium keine fremden Metallsulfide $\mathrm{ab}$

8. Nach Ausfällen mit Ammoniak geht |Kupfer, Cadmium, Zink, kein fremdes Metall in das Filtrat über $\}$ Silber.

9. Die Nitratlösung wird durch Barium-
nitrat nicht gefällt Schwefel, Selen.

10. Ebensowenig durch Ammoniummolybdat\} Phosphor usw.

11. Die verdünnte Nitratlösung darf durch Behandlung mit schwefliger Säure keine Trübung erfahren

12. Die Flektrolyse der Jı̈̈sung darf an der Platinanode kein fremdes Superoxyd ergeben

Durch einzelne dieser Proben, denen sich auch die Spektralreaktionen anschließen, wird man schon bei Anwendung weniger Gramme leicht feststellen können, ob ein vorliegendes Wismutmetall stark oder schwach verunreinigt, d. h. nach dem technischen Sprachgebrauch unrein oder scheinbar rein ist. Zur näheren Charakterisierung dient die quantitative Analyse. 
Stark verunreinigtes Wismutmetall wird man (bei Anwendung von 2 bis $10 \mathrm{~g}$ ) nach den bewährten Methoden der technischen Handbücher ${ }^{1}$ analysieren.

Zur Aufsuchung und Bestimmung der Verunreinigungen im nominell reinen Wismut ist aber eine größere Metallmasse und eine gesteigerte Sorgfalt aufzuwenden. Das folgende Verfahren hat sich dazu als durchführbar und zweckmäßig erwiesen.

\section{Quantitative Analyse von nominell reinem Wismat.}

a) Auflösung und Kristallisation.

$100 \mathrm{~g}$ des in Brocken oder Stäben vorliegenden Metalls werden in einem Quarzkolben nach und nach mit $250 \mathrm{ccm}$ chlorfreier Salpetersäure (spez. Gew. 1.40) übergossen, wobei, anfangs ohne äußere Erwärmung, eine vollständige Auflösung des Metalles erfolgt. Nach dem Erkalten liegt entweder eine klare oder eine getrübte Lösung vor, welche bei dem „Impfen“ den größten Teil des Wismutnitrats rasch zur Abscheidung gelangen läßt.

Ohne einen etwaigen unlöslichen Rückstand abzufiltrieren, ist es vorteilhaft, die Kristallisation möglichst bei langsamem Erkalten (über Nacht) aus warmer Lösung vor sich gehen zu lassen, weil sich dann große festhaftende Kristalle von Wismutnitrat am Boden des Gefäßes bilden, von welchen man die Mutterlauge leicht abogießen kann (Kristalle I). Die letztere, auf $1 / 4$ eingedampft, liefert dit; Kristalle II, die Mutterlauge davon die Kristalle III usw. Fndlich erhält man wenige Kubikzentimeter einer meist grün gefürbten „letzten Mutterlauge“, in welcher die Hauptmenge der löslichen Verunreinigungen neben wenigen Grammen Wismutnitrat vorhanden ist. Nach dieser Vorbehandlung kommt es nun darauf an, die abgeschiedenen Kristallfraktionen in reines Wismutnitrat überzuführen.

Die bei der Auflösung des Metalles rückständigen oder niedergeschlagenen Teile der Verunreinigungen sind zumeist nit den Kristallen I vereinigt. Diese Hauptkristallfraktion von etwa $250 \mathrm{~g}$ wird mit der Hälfte ihres ('ewichtes $8 \%$ iger Salpetersäure 1 Stunde lang erwärmt. Für den F'all einer erneuten I'rübung (von Zinnsäure) läßt man den Niederschlag bei Zimmertemperatur

1 Z. B. LUNGE, Chemisch-Teclin. Untersuchungsmethoden II, 278; Moser, Die Bestimmung des Wismuts usw. 
(über Nacht) sich absetzen und filtriert die Lösung dann (wenn nötig wiederholt) durch ein gewöhnliches Filter, auf welchem der Niederschlag (oder etwaiger Lösungsrückstand) gesammelt und ohne Auswaschen zu spätcrer Verarbeitung aufgehobenwird (Niederschlag A).

Das völlig klare Filtrat wird nach S.245 unter Zusatz des gleichen Gewichtes konzentrierter Salpetersäure (1.40) auf $0^{\circ}$ abgekühlt. Die nach dem Impfen und Agitieren nach längerer Zeit ausgeschiedenen kleinen Kristalle, über einem Platinkonus stark abgesaugt und mit eiskalter konzentrierter Salpetersäure gewaschen, betragen gegen $150 \mathrm{~g}$ der Verbindung $\mathrm{Bi}\left(\mathrm{NO}_{3}\right)_{3}+5 \mathrm{H}_{2} \mathrm{O}$, welche nun als rein betrachtet und beseitigt werden kann.

In der gleichen Weise werden auch die weniger reinen Fraktionen (Kristalle I, II, III usw.) eventuell nach ihrer Vereinigung, auf reines Wismutnitrat verarbeitet. Falls diese Fraktionen bei dem Erwärmen mit $8 \%$ iger Salpetersäure keine Trübung ergeben, kann man sie auch durch Erwärmen in der von der Hauptfraktion des reinen Nitrats abfiltrierten Mutterlauge lösen, und erhält dann bei der Abkühlung auf $0^{0}$ oder $-10^{\circ}$ eine zweite Fraktion gereinigter Nitratkristalle, welche wieder beseitigt werden kann usw.

Die Reinigung des Wismutnitrats durch Kristallabscheidung aus den durch Eindampfen konzentrierten, stark verunreinigten und vom Kupfernitrat meist grün gefärbten Mutterlaugen kann durchgeführt werden, solange die farblosen Nitratkristalle kein Blei enthalten. Dieser Fall kann eintreten, wenn der Bleigehalt in der Mutterlauge auf etwa $1 \%$ des Wismuts gestiegen ist. (Man prüft die abgeschiedenen Kristalle nach der Überführung in Chlorid mit Alkohol und Schwefelsäure und kann den etwa ermittelten Bleigehalt später in Rechnung bringen; die Prüfung auf Blei kann auch auf elektrolytischem Wege geschehen.)

Die dann noch verbleibende grünliche Mutterlauge von sehr geringem Volumen wird mit der bei den anfänglichen Kristallisationen erhaltenen letzten Mutterlauge vereinigt. Man verdampit dann den Säureüberschuß so weit, da $B$ bei dem Erkalten Kristallisation erfolgt und fügt ein wenig $8 \%$ ige Salpetersäure dazu. Ein bei der Auflösung bleibender Rückstand wird abtiltriert, mit der gleichen Säure gewaschen und zum unlöslichen Rückstand A gefügt. Das Filtrat wird als unreine Mutterlauge B unabhängig vom Rückstand $\mathrm{A}$ analysiert. 

b) Analyse des ungelösten Rückstandes A.

Der von verdünnter Salpetersäure nicht gelöste Rückstand des Wisnuts kann silikathaltige Schlackenteile, kohlige Substanz, Platinmetalle, Gold, Silberverbindungen, Zinnsäure, Antimonsäure, Arsensäure, Phosphorsäure usw. in Verbindung mit Wismut, Spuren von Kupfer, Blei, Eisen usw. enthalten.

Die Analyse nimmt je nach der Natur des Rückstandes, für welche schon die Färbung (schwarz oder weiß) einen Anhalt gibt, verschiedene Formen an gemäb der folgenden Operationen, welche nacheinander zur Aufschließung dienen können.

1. Extraktion mit konzentrierter Salzsäure. In Lösung gehen Arsen, Antimon (Zinn), Wismut, Silber, Kupfer, Blei, Eisen usw.

2. Extraktion mit Königswasser. In Lösung gehen Gold, Platinmetalle usw.

3. Schmelzen mit Soda und Schwefel zur Aufschließung der Metazinnsäure usw.

4. Aufschließen der Silikate durch Schmelzen mit Soda.

Wir begnügen uns hier mit Andeutungen über die Verarbeitung des salzsauren Auszuges (1). Man verdünnt denselben, fällt mit Schwefelwasserstoff und behandelt die Sulfide mit gelber Schwefelnatriumlösung; die darin unlöslichen Sulfide können in Salpetersäure gelöst und zugleich mit dem entsprechenden Material der ,,unreinen Mutterlauge B“ analytisch getrennt werden; ebenso ist es zweckmäßig, die aus dem gelben Sulfidfiltrat mit Sänre gefällten Sulfide von Arsen, Antimon und Zinn behufs der Trennung mit den analogen Sulfiden aus der Mutterlauge zu vereinigen.

Das gleiche gilt von der unter 3. erwähnten Aufschließung mit Soda und Schwefel, welche vorzugsweise Zinnsulfıd ergeben wird:

c) Analyse der unreinen Wismutnitrat-Mutterlauge B.

1. Die mit verdünnter Salpetersäure vermischte Mutterlauge wird mit sehr wenig Salzsäure versetzt zur Fällung des Silbers, solange noch ein Niederschlag von $\mathrm{AgCl}$ ausfällt. Nach längerem Stehen wird filtriert. Der mit verdünnter Salpetersäure gewaschene Niederschlag wird mit Ammoniak extrahiert (zur Beseitigung von Wismut und Blei), im Filtrat mit Salpetersäure als gereinigtes Chlorsilber aufs neue gefällt und in bekannter Weise isoliert.

2. Das saure Filtrat von 1. wird zunächst für sich, und der Rückstand dann wiederholt mit Salzsüure verdampft zur Ver- 
treibung der Salpetersäure. Der in etwas Salzsäure gelöste Chloridrückstand wird durch allmählichen Zusatz von viel Wasser in Oxychlorid (I) übergeführt; dasselbe wird abfiltriert, mit Wasser gewaschen und getrocknet. Das Filtrat wird nochmals vorsichtig eingedampft und der kleine Chloridrückstand abermals mit Wasser als Oxychlorid II gefällt.

3. Das salzsäurehaltige Filtrat von 2., welches fast ganz vom Wismut befreit ist, wird bei Zimmertemperatur durch kurzes Finleiten mit Schwefelwasserstoff gesättigt. Der schwarze Sulfidniederschlag wird abfiltriert, mit Wasser gewaschen, in Salpetersäure gelöst und, wie bekannt, durch Eindampfen der Lösung mit Schwefelsäure usw. auf Blei und nötigenfalls neben dem Kupfer auch auf Cadmium usw. geprüft. Häufig wird es genügen, den überwiegend Kupfersulfid enthaltenden Niederschlag durch Glühen in Oxyd überzuführen und dasselbe zu wägen. Man löst dasselbe in verdünnter Salpetersäure oder Ameisensäure, wobei Gold, Platin usw. zurückbleiben würden, und bestimmt das Kupfer im Filtrat nach dem Übersättigen mit Ammoniak kolorimetrisch. Die Differenz mit der Wägung als Oxyd würde der Verunreinigung im Sulfid entsprechen.

4. Das vom Kupfersulfid befreite salzsaure Filtrat wird nunmehr in der Wärme aufs neue mit Schwefelwasserstoff behandelt. Das gefällte Arsenpentasulfid kann auf gewogenem Filter gesammelt werden. Ebenso gelangt hier als Sulfid das etwa in Spuren vorhandene Platin usw. zur Abscheidung, welches nach der Filtration geglïht und als gelber Platinsalmiak identifiziert wird.

5. Das zur Entfernung von Schwefelwasserstoff erwärmte Filtrat von 4. wird oxydiert und mit Ammoniak übersättigt zur Fällung von Eisen, Mangan und Aluminium, welche gewöhnlich nur in Spuren vorhanden sind. Man wägt den geglühten Niederschlag und kann darin das Fisen nach dem Auflösen in konzentrierter Salzsäure (nach HürtwnR) leicht kolorimetrisch bestimmen.

6. In das ammoniakalische erwärmte Filtrat von 5. wird Schwefelwasserstoff geleitet, wobei Nickel (Kobalt) zugleich mit Zink gefällt wird. Der Niederschlag wird abfiltriert und gewaschen. Die Trennung bewirkt man durch Extraktion mit verdünnter Salzsäure, wobei Nickel als Sulfid zurückbleibt, welches sich nach bekannter Weise leicht in wägbares Oxyd überführen läBt. Nachdem das saure Filtrat wieder ammoniakalisch gemacht wurde, kann das Zink wieder als Sulfid gefällt und auf gewogenem Filter gravimetrisch bestimmt werden. 
Enthält das Sulfia noch Nickel, so ist eine wiederholte reinigende Fällung des Zinksulfids aus ameisensaurer Lösung leicht durchführbar.

7. Das ammoniakalische Filtrat von 6., welches vom Nickelund Zinksulfid getrennt wurde, sollte bei dem Verdampfen und Glühen keinen Rückstand hinterlassen (außer den Spuren der Metallverbindungen, welche den analytischen Fehlergrenzen entsprechen [Nickel usw.] oder aus den Reagentien und den Gefäßen stammen). Ist ein solcher aennoch beachtenswert, so hönnte er aus Verbinaungen von Leichtmetallen bestehen, welche im nominell reinen Wismut aber kaum vorkommen.

8. Das nach 2. durch Wasser gefällte Wismutoxychlorid I und II enthält gewöhnlich den größten Teil des vorhandenen Bleies. Zu seiner Bestimmung wird die trockene Substanz in dem doppelten Gewicht konzentrierter Salzsäure gelöst unter Zusatz der 10 fachen Gewichtsmenge absoluten Alkohols. Geringe Gelbfärbung würde dabei eine Spur Kupfer anzeigen, welche sich kolorimetrisch abschätzen läßt. Die Lösung wird nach Hinzufügen von 0.1 Gewichtsteilen Schwefelsäure einer 24 stündigen Ruhe überlassen. 1)as dabei abgeschiedene Bleisulfat wird auf gewogenem Filter gesammelt, mit Alkohol gewaschen, bei $100^{\circ}$ getrocknet und gewogen. Es ist noch auf einen Silbergehalt zu prüfen.

9. Das saure alkoholische Filtrat von 8. enthält das Wismutchlorid. Neben einer bereits abgeschätzten Spur Kupfer könnte auch noch ein wenig Arsen vorhanden sein. Man prüft darauf, indem man das Filtrat bei Zimmertemperatur mit einem Drittel seines Volumens konzentrierter Salzsäure versetzt und mit Schwefelwasserstoff sättigt, wobei das Wismut in Lösung bleibt. Hin etwa entstehender Sulfidniederschlag, mit Salzsäure gewaschen, wird in bekannter Weise näher untersucht.

Der. kundige Leser wird finden, daß die vorstehende Anleitung eher zu einfach als zu detailliert ist, daß sie mit Vorliebe dic gröbere Unterscheidung in Anwendung bringt und keinen Wert darauf legt, die Trennung der Verunreinigungen voneinander mit der Genauigkeit von Präzisionsanalysen durchzuführen; dies wäre sehr mühevoll. Hier kommt es quantitativ mehr auf die summarische Massenbestimmung aller Verunreinigungen an, welche qualitativ mit aller Schärfe zu definieren sind. Ist die Masse sehr klein $(1 \mathrm{mg}$ oder dgl.), so wird der Fehler bei der Bestimmung einzelner Komponenten oft erheblich über $10 \%$ des richtigen Wertes hinausgehen. 
In diesem Falle können die schärfsten Bestimmungsmethoden zweckmäßig sein.

Es soll endlich besonders betont werden, daß hier der Einfachheit wegen die selteneren sowie die nichtmetallischen Verunreinigungen des Wismuts unberücksichtigt geblieben sind; das Buch von Mosen gibt hier die nötigen Anleitungen.

\section{Chemische und physikalische Charakterisierung von Wismutmetall. \\ 1. Die chemische Analyse.}

In der vorstehend beschriebenen Form wurde die Analyse zur Charakterisierung mehrerer aus dem deutschen Handel bezogener Proben von angeblich reinem Wismutmetall benutzt. Die Werte für die gefundenen Verunreinigungen sind in der folgenden Tabelle zusammengestellt.

Tabelle 1.

Verunreinigungen in Prozent.

\begin{tabular}{|c|c|c|c|c|c|c|c|}
\hline & \multicolumn{3}{|c|}{ von Schuchardt } & \multicolumn{2}{|c|}{$\begin{array}{l}\text { von Hartmann } \\
\& \text { Braun }\end{array}$} & \multicolumn{2}{|c|}{ von Kahlbaum } \\
\hline & 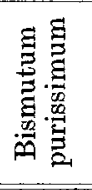 & 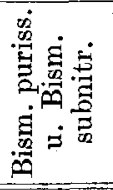 & 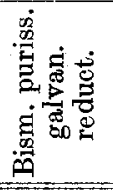 & 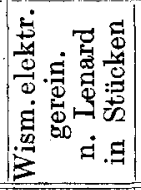 & 离 & 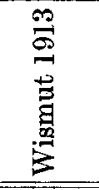 & 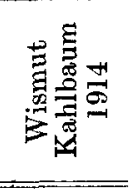 \\
\hline Probe Nr. & 1 & 2 & 3 & 4 & 5 & 7 & 8 \\
\hline $\begin{array}{l}\text { Silber . : } \\
\text { Kupfer : } \\
\text { Blei . : } \\
\text { Eisen : : } \\
\text { Zink. : } \\
\text { Nickel . : } \\
\text { Zinn. : } \\
\text { Antimon : } \\
\text { Platin . } \\
\text { Kohlenstoff } \\
\text { Kieselsäure }\end{array}$ & $\begin{array}{l}0.012 \\
0.02 \\
0.006 \\
\text { Spur } \\
0.012 \\
0.003 \\
0.13 \\
\text { Spur } \\
\\
\text { Spur }\end{array}$ & $\begin{array}{c}0.0025 \\
0.0306 \\
\text { Spur } \\
\text { Spur } \\
\\
0.0094 \\
0.057 \\
\text { Spur }\end{array}$ & $\begin{array}{l}0.0005 \\
0.01 \\
\text { Spur } \\
0.01\end{array}$ & $\begin{array}{l}0.20 \\
0.002 \\
0.01\end{array}$ & $\begin{array}{c}0.254 \\
0.0013 \\
\text { Spur } \\
\text { Spur } \\
\\
\text { ca. } 0.02 \\
\\
0.021\end{array}$ & $\begin{array}{l}0.026 \\
0.0024 \\
0.035 \\
\text { Spur }\end{array}$ & $\begin{array}{c}\text { Spur } \\
0.001 \\
\text { Spur } \\
\text { Spur }\end{array}$ \\
\hline Summe & 0.2 & 0.1 & 0.03 & 0.25 & 0.3 & 0.06 & $\overline{\mid ~ u n t e r ~} 0.01$ \\
\hline $\begin{array}{l}\text { Reinigungs- } \\
\text { stufe }\end{array}$ & II & II & III & II & II & III & IV \\
\hline
\end{tabular}

Aus der Zusammenstellung sind die nachstehenden Tatsachen besonders bemerkenswert.

1. Gemäß der Gesamtverunreinigung, welche nicht über $0.3 \%$ hinausgeht, gehören die Proben der II., III. und IV. Reinigungsstufe an.

2. Alle Proben enthalten leicht bestimmbare Mengen metal- 
lischer Verunreinigungen, unter welchen gewöhnlich Silber, Blei und Lupfer, bisweilen auch Zinn, Nickel, Zink und Platin vertreten sind.

3. Auch die Proben von elektrolytischem Wismut waren nicht rein; zwei davon (4. und 5.) enthielten Platin und Silber, die dritte (3.) neben den üblichen Spuren Blei und Kupfer auch noch Zink. Das Platin ${ }^{1}$ stammt anscheinend aus den Kathoden, das Zink aus dem Elektrolyten, dessen Natur uns nicht bekannt war.

Von Interesse ist die Feststellung, daß bei der elektrolytischen Übertragung das Silber häufig mit dem Wismut gefällt wird, und daB auch das Blei als kathodische Verunreinigung nicht leicht ganz vermieden werden kann.

4. Das Wismut 2 unterscheidet sich vom Metall 1 und 2 vorteilhaft durch die geringe Anzahl der Verunreinigungen und durch die völlige Löslichkeit in Salpetersäure, da es frei von Zinn und Antimon ist. Zur weiteren Reinigung eignet sich dies Metall daher besonders gut.

5. Das Wismut „Kahlbaum“ 1914 (Probe 8) wurde auf Veranlassung der Reichsanstalt aus gereinigtem kristallisierten Nitrat dargestellt; es hat sich am reinsten erwiesen und gehört der IV. Reinigungsstufe an (maximale Verunreinigung zum Wismut wie $1: 10^{4}$ ) gleich den früher charakterisierten nominell reinen Metallen ${ }^{2}$ Zink, Blei, Cadmium, Zinn aus der gleichen Bezugsquelle Kahlbaum.

Auch in dem reinsten im technischen Betriebe hergestellten Wismutmetall (Probe 8) sind also immerhin noch Verunreinigungen nachweisbar, obwohl hier das vorstehend empfohlene präparative Nitratverfahren angewandt worden ist.

Werden die Operationen aber mit besonderer Sorgfalt ausgeführt, so gelingt es, noch reineres Metall zu gewinnen. Solche Versuche sind in der Reichsanstalt durchgeführt und haben Wismutproben ergeben, welche wohl zur V. oder VI. Reinigungsstufe gehören, da ihre Verunreinigungen nicht mehr zu erkennen waren. Es scheint kein Grund vorhanden, weswegen nicht gelegentiich so reine Produkte auch in größerem Maßstabe hergestellt werden könnten.

\section{Der schmelzpunkt.}

Der Schmelzpunkt gilt mit Recht als eine der wichtigsten eindeutigen Konstanten elementarer Metalle. Eine Abhängigkeit von

1 Auf eine Anfrage bei Hartmann \& Braun wurde mitgeteilt, daB nur Wismutanoden, nicht aber Platinanoden verwendet werden.

${ }^{2}$ Vgl. Myruus, $Z$. anorg. ('hem. 1. c. 
etwaigen allotropen Modifikationen ist bei dem Wismut von Coren und Moesveld ${ }^{1}$ zwar vermutet, aber bisher nicht nachgewiesen worden: starke Abweichungen bedingen aber die Verunreinigungen.

Über den Schmelzpunlit des Wismuts liegen viele verschiedene Angaben vor, welche von 262 bis $277^{\circ}$ wechseln. In der auf S. 248 abgedruckten Tabelle der Schmelzpunktserniedrigungen durch Fremdstoffe sind auch die Beobachtungen über den Schmelzpunkt des angewanaten Wismuts angeführt. Man hat für die Versuche wohl selten ganz reines, sondern meistens verunreinigtes Metall aus dem Handel benutzt. In vielen Fällen kam es den Beobachtern auch mehr auf Temperaturdifferenzen als auf absolute Temperaturbestimmungen an; die Angaben beziehen sich dann zwar auf die Grade der Meßapparate aber nicht auf Celsiusgrade. Nur so sind z. B. die Angaben $277^{\circ}$ (ATEN 1905) und 262 (Voges 1914) erklärbar. Bei dieser Unsicherheit erscheint eine Revision des Schmelzpunktes vom Wismut durchaus erforderlich.

Zu den Bestimmungen wurde das auf, Veranlassung der Reichsanstalt aus gereinigtem Nitrat hergestellte Wismut „Kahlbaum 1914“ benutzt, welches wenigstens der vierten Reinigungsstufe angehört mit Verunreinigungen von weniger als $1 / 100 \%$; bei der Analyse konnte nur eine Spur Kupfer im Betrage von $0.001{ }^{0} i_{0}$, sowie geringe Spuren von Blei und Eisen nachgewiesen werden.

Aus den Vorversuchen der Herren Hofrmann und Moesler ergab sich die Schmelztemperatur wesentlich unterhalb $272^{\circ}$. Herr Direktor Holborn führte die genaue Bestimmung unter AnschluB an die jetzt maßgebende thermodynamische Thermometerskala aus und macht darüber die folgenden Angaben:

„Für den Schmelz- und Erstarrungspunkt des übergebenen Wismuts wurde der Wert $271.0^{\circ}$ gefunden. Die Menge von $800 \mathrm{~g}$, die unter Paraffin in einem Glasrohr geschmolzen wurde, war etwas klein, so daß sich die einzelnen Bestimmungen etwas mehr unterscheiden wie bei der sonst gebräuchlichen Metallmenge. ${ }^{2}$ Doch überschreiten die Abweichungen der Einzelmessungen vom Mittel nicht $0.15^{\circ}$. Schmelzpunkt und Erstarrungspunkt zeigten keinen Unterschied. Der gefundene Wert stimmt mit der Zahl überein, die Adams und JoHnston (Am. Journ. of Sc. [4] 33 (1912), 545 angeben. ${ }^{3}$ Sie benutzten Bakers Metall.“

\footnotetext{
1 Coher und Monsverd, Zeitschr. phys. Chem. 85 (1913), 428.

2 Vgl. MyuJus, Z. anorg. Chem. 74 (1912), 424.

3 Vgl. Z. anorg. Chem. 72 (1911), 20.
} 
Bei der großen Sicherheit der Temperaturmessung und der verschwindend geringen Verunreinigung wird dieser Wert als der wahre Schmelzpunkt des Wismuts anzunehmen sein. Der Schmelzpunkt von Metall, welches durch vielfache Kristallisation aus dem Schmelzfluß noch weiter gereinigt war, zeigte keine merkliche Veränderung; auch hier fand Herr MoELLFr bei Versuchen in kleinerem Maßstabe $271.0^{\circ}$. Anch die Bestimmungen von

$$
\begin{aligned}
& \text { Vogre . . . . . . } 271^{0} 1906 \\
& \text { Fugink . . . 271.50 } 1908 \\
& \text { BARLOW . . . } 271.2^{\circ} 1911 \\
& \text { Petrenko . . . } 271^{\circ} 1915
\end{aligned}
$$

ergaben nahezu die gleichen Werte.

\section{Der elektrische Widerstand.}

Schon 1862 beschäftigten sich Matrhiessen und v. Bose ${ }^{1}$ mit dem elektrischen Leitvermögen yon Wismutdrähten und dessen Beiinflussung durch die Temperatur. Spätere Beiträge wurden von RIGHI ${ }^{2}$ und von AUBEL ${ }^{3}$ geliefert. Mit besonderer Sorgfalt hat LenA RD ${ }^{4} 1890$ den elektrischen Widerstand gepreßter Drähte aus Wismutmetall untersucht; er findet ihn abhängig einerseits vom Reinheitsgrade und andererseits von der Preßtemperatur. Aus den von uns durch chemische Analyse charakterisierten Wismutproben (S. 259) hat Herr Werner bei verschiedenen Temperaturen Drähte von $1 \mathrm{~mm}$ Dicke durch Pressen hergestellt und deren elektrischen Widerstand sorgfältig bestimmt. Unter den vergleichbaren Proben befand sich auch nominell reines Wismut von Hartmann \& Braun, welches nach den Angaben von LENARD durch elektrolytische Übertragung in Nitratlösung hergestellt und von der Reichsanstalt in mehreren Sendungen sowohl in Stücken wie als fertiger Draht bezogen wurde.

Außerdem wurde Wismutmetall untersucht, welches nach der chemischen Reinigung in der Reichsanstalt noch wiederholt aus dem Schmelzfluß umbristallisiert worden war. Über die vielen unter mannigfachen Bedingungen ausgeführten Bestimmungen wird Herr Werner an anderer Stelle ausführlich berichten; die folgenden uns übermittelten Angaben boziehen sich ausschließlich auf die vergleichbaren Werte des spezifischen Widerstandes bei $22^{\circ}$ für die PreBtemperatur $195^{\circ}$.

1 Matthessen und v. Bose, Pogg. Ann. 115 (1862), 353.

2 RIGH, Nuov. Cim. 31 (1885), 42.

3 AUber, Ann. Chim. Phys. [6] 18 (1889), 433.

4 Lenard, Wied. Ann. 39 (1890), 638. 
Tabelle 2.

\begin{tabular}{|c|c|c|c|c|c|}
\hline $\begin{array}{c}\text { Probe } \\
\text { Nr. }\end{array}$ & Herkunft & Bezeichnung & $\begin{array}{l}\text { Reini- } \\
\text { gungs- } \\
\text { stufe }\end{array}$ & $\begin{array}{c}\text { Prozent } \\
\text { an Verun- } \\
\text { reinigung }\end{array}$ & $\begin{array}{l}\text { Spez. } \\
\text { elektr. } \\
\text { Wider- } \\
\text { stand }\end{array}$ \\
\hline 1 & Dr. Theod. Schuchardt & Bismutum purissimum & II & 0.2 & 1.30 \\
\hline 2 & ", & $\begin{array}{l}\text { Bism. puriss.e Bism. sub- } \\
\text { nitrico. }\end{array}$ & II & 0.1 & 3.09 \\
\hline 3 & , & $\begin{array}{l}\text { Bismut. puriss. galvan. } \\
\text { reduct. }\end{array}$ & III & 0.03 & 1.33 \\
\hline 4 & Hartmann \& Braun & $\begin{array}{l}\text { Wismut elektr. gereinigt } \\
\text { n. L, in Stücken, } \\
\text { Sendung I }\end{array}$ & II & 0.25 & \\
\hline 5 & , & $\begin{array}{l}\text { Wismut elektr. gereinigt } \\
\text { n. L. als Draht, Send.I }\end{array}$ & II & 0.3 & 1.29 \\
\hline 6 & , & $\begin{array}{c}\text { Wismut elektr. gereinigt } \\
\text { n. L. in Stücken, Send.II }\end{array}$ & IV & 0.01 & 1.24 \\
\hline 7 & C. A. F. Kahlbaum & Wismut 1913 & III & 0.1 & 1.22 \\
\hline 8 & , & Wismut „Kahlbaum “ 1914 & IV & unter 0.01 & 1.21 \\
\hline $\mathbf{9}$ & P. T. Reichsanstalt & $\begin{array}{l}\text { Wism, aus reinem krystall. } \\
\text { Nitrat } 1914\end{array}$ & über IV & & 1.19 \\
\hline 10 & , & $\begin{array}{l}\text { Wism. von Probe } 7 \text {, aus } \\
\text { dem Schmelzflu } B \text { kryst. }\end{array}$ & über IV & & 1.20 \\
\hline 11 & ", & $\begin{array}{l}\text { Wism. von Probe } 8 \text { aus } \\
\text { dem SchmelzfluB krist. }\end{array}$ & über IV & & 1.20 \\
\hline
\end{tabular}

,Sieht man von den Proben 1 bis 3 gleicher Herkunft, die ein ganz abweichendes Verhalten anfweisen, $a b$, so nimmt in der Tat, wie schon RIGHI angibt, der spezifische Widerstand mit zunehmender Reinheit des Wismuts ab. Die genannten drei Proben dagegen zeigen einen wesentlich größeren Widerstand, als nach dem Ergebnis der Analyse za erwarten ist. Bemerkenswert ist der besonders große Widerstand von Probe 2, welche aus gefälltem basischen Wismutnitrat erhalten war. Sie hat im Gegensatz zu allen übrigen untersuchten Wismutproben einen negativen Temperaturkoeffizienten und zeichnet sich, wie auch Probe 1, durch Verunreinigungen aus der Zinngruppe aus.

Nach den alten Versuchen von MatrHiessen ist anzunehmen, daß die chemische Natur einer Verunreinigung von großem Einfluß auf die von ihr hervorgebrachte Widerstandsvermehrung ist, und RIGHI ${ }^{1}$ hat bereits darauf hingewiesen, daß besonders ein Zinngehalt den elektrischen Widerstand des Wismuts stark erhöht.

Die in der Reichsanstalt gewonnenen Wismutproben, welche auf dem Wege der Kristallisation gereinigt warer, zeigten den

1 RIaH, Fortschr. d. Phys. 40 II (1884), 733. 
geringsten spezifischen Widerstand, und zwar war Probe 9 (Kristallisation als Nitrat aus wässeriger Lösung) in naher Übereinstimmung mit den Proben 10 und 11 (Kristallisation des Metalls aus dem Schmelzfluß). Diese Werte sind um mehrere Prozent niedriger als der Wert 1.24 der Probe 6 Send.II, welche bei Hartmann \& Braun sorgfältig elektrolytisch gereinigt worden war. Die Vermutung, daß hier noch Verunreinigungen nachweisbar seien, hat sich bestätigt; eine mit $43 \mathrm{~g}$ des Metalls ausgeführte Analyse ergab:

$$
\begin{aligned}
& \text { Platin . . . . } 0.0032 \% \\
& \text { Silber . . . . . } 0.0065 \% \\
& \text { Eisen . . . . Spuren }
\end{aligned}
$$

Damit ist gezeigt, daß die elektrolytische Reinigung des Wismuts schwieriger durchführbar ist als der chemische Weg, welcher zu höheren Reinigungsstufen führt. Andererseits muß aber bemerkt werden, daß der in der Reichsanstalt für das Wismut in J)rahtform festgestellte Wert 1.20 nicht unerheblich urößer ist als die Werte 1.09 his 1.16 (je nach der Preßtemperatur), welche LENARD 1890 angibt.“

\section{Ergebnis der Untersuchung.}

1.Die präparative Reinigung des Wismuts geschieht am rationellsten durch Kristallisation, und zwar a) des normalen Nitrats $\mathrm{Bi}\left(\mathrm{NO}_{3}\right)_{3} \div 5 \mathrm{H}_{2} \mathrm{O}$ aus wässerig saurer Iösung und b) des Metalls aus dem Schmelzfluß.

2. Die Nitratkristallisation bewährt sich auch als Grundlage für die analytische Bestimmung kleiner Verunreinigungen; ein Gang für die Cntersuchung nominell reinen Wismuts wird beschrieben.

3. Die Kristallisationsmethode (der chemische Weg) führt leichter zu reinem Wismut als der elektrolytische Weg.

4. Eine Anzahl nominell reiner Wismutproben verschiedener Herkunft wird durch Analysen chemisch charakterisiert.

5. Der Schmelzpunkt des elementaren Wismutmetalls wrrd zu $271^{\circ}$ festgestellt.

6. Der elektrische Widerstand von reinem Wismutiraht (Preßtemperatur $195^{\circ}$ ) ergibt sich zu $1.20 \mathrm{Ohm}$ zum Unterschiede von höheren Werten, welche bei verunreinigten Wismutproben gefunden werden.

Charlottenburg, März 1916.

Bei der Redaktion eingegangen am 8. Mai 1916. 